\title{
Prodelphinidins isolated from Chinese bayberry leaves induces apoptosis via the p53-dependent signaling pathways in OVCAR-3 human ovarian cancer cells
}

\author{
YU FU ${ }^{1,2}$, XINGQIAN YE ${ }^{1}$, MALCOLM LEE ${ }^{2}$, GARY RANKIN $^{3}$ and YI CHARLIE CHEN ${ }^{2}$ \\ ${ }^{1}$ College of Biosystems Engineering and Food Science, \\ Fuli Institute of Food Science, Zhejiang University, Hangzhou, Zhejiang 310058, P.R. China; \\ ${ }^{2}$ College of Science, Technology and Mathematics, Alderson Broaddus University, \\ Philippi, WV 26416; ${ }^{3}$ Department of Pharmacology, Physiology and Toxicology, \\ Joan C. Edwards School of Medicine, Marshall University, Huntington, WV 25755, USA
}

Received April 27, 2016; Accepted December 2, 2016

DOI: $10.3892 / \mathrm{ol} .2017 .5813$

\begin{abstract}
Chinese bayberry leaves are rich in prodelphinidins. Since the isolation and purification of prodelphinidins is difficult, the association between the degree of prodelphinidin polymerization and their anti-carcinogenic activity remains ambiguous. The cytotoxic and apoptotic activities of prodelphinidin Chinese bayberry leaf extracts (PCBLs), oligomeric proanthocyanidins (OPAs) and polymeric proanthocyanidins (PPAs), isolated by normal-phase preparative high-performance liquid chromatography were investigated in OVCAR-3 human ovarian cancer cells. The PCBLs, OPAs and PPAs inhibited cancer cell growth and induced apoptosis via the caspase-dependent pathway. Apoptosis was triggered through the intrinsic pathway by upregulating the expression of several B-cell lymphoma-2 (Bcl-2) family proapoptotic proteins, including p53-upregulated modulator of apoptosis (PUMA), Bcl-2-associated X protein and Bcl-2-associated agonist of cell death, and by downregulating the antiapoptotic protein Bcl-extra large. Apoptosis was also triggered through the extrinsic pathway via the upregulation of death receptor 5 (DR5) and Fas expression. In addition, OPAs and PPAs induced caspase-dependent apoptosis at least partially through the inhibition of the protein kinase B signaling pathway. The knockdown of $\mathrm{p} 53$ by specific small interfering RNA resulted in the depletion of p53, and inhibited the OPA and PPA
\end{abstract}

Correspondence to: Dr Yi Charlie Chen, College of Science, Technology and Mathematics, Alderson Broaddus University, 101 College Hill Road, Philippi, WV 26416, USA

E-mail: chenyc@ab.edu

Dr Xingqian Ye, College of Biosystems Engineering and Food Science, Fuli Institute of Food Science, Zhejiang University, 866 Yuhangtang Road, Hangzhou, Zhejiang 310058, P.R. China

E-mail: psu@zju.edu.cn

Key words: Myrica rubra, leaves, prodelphinidins, apoptosis, ovarian cancer treatment-induced increases in $\mathrm{p} 53$, which led to a decrease in the expression of p21, DR5, Fas, PUMA and phosphatase and tensin homolog proteins. These observations demonstrate that p53 is a mediator of OPA and PPA-induced apoptosis in OVCAR-3 cells. The PPAs exhibited stronger anti-proliferative and pro-apoptotic activities compared with OPAs and PCBLs. These results suggest that PCBLs, OPAs and PPAs may be useful for the treatment of ovarian cancer.

\section{Introduction}

The use of botanicals for the prevention of various diseases has been a subject of interest, and phytochemicals have been indicated to be useful as protective agents against certain types of cancer, but the investigation of proanthocyanidins has been limited (1).

Proanthocyanidins, also termed condensed tannins, are a group of oligomers or polymers of flavan-3-ols. They are ubiquitous and represent the second most abundant group of natural phenolics after lignin (2). Oligomers and polymers of proanthocyanidins can be widely found in the plant kingdom in fruits, berries, seeds, flowers and leaves (3). The proanthocyanidins that exclusively consist of epicatechin units are termed procyanidins and are the most abundant type of proanthocyanidins in plants. The less common proanthocyanidins that contain epigallocatechin subunits are termed prodelphinidins (1).

Chinese bayberry (Myrica rubra) is a subtropical evergreen fruit tree that is widely grown in southern China $(4,5)$. Chinese bayberry leaves are rich in prodelphinidins $(6,7)$. Few studies have been conducted to assess the anticancer effects of prodelphinidins extracted from Chinese bayberry leaves (PCBLs), and the molecular mechanisms underlying the anticancer effect of PCBLs has been minimally investigated in human cancer cells. As it is difficult to separate prodelphinidins according to their degree of polymerization and isolate the pure standard from mixtures including other polyphenols, the association between the degree of polymerization and anti-carcinogenic activity remains ambiguous (8). 
Apoptosis is triggered through two major pathways: The intrinsic pathway and the extrinsic pathway (9). Apoptosis serves a major role in establishing a natural balance between cell death and renewal by destroying excess, damaged or abnormal cells (3). As cancer may result from uncontrolled cell proliferation and dysregulation of apoptosis, the induction of apoptosis is a highly desirable goal in developing preventive strategies for cancer control (10).

The present study aimed to isolate and characterize PCBLs, and investigate the mechanism of their anticancer effect. The study focused on caspase-dependent apoptosis, particularly the two major apoptotic pathways (intrinsic and extrinsic pathway) and p53-associated apoptosis, to determine whether PCBLs exhibited anticancer properties in OVCAR-3 cells.

\section{Materials and methods}

Preparation of PCBLs, oligomeric proanthocyanidins (OPAs) and polymeric proanthocyanidins (PPAs). PCBLs, OPAs and PPAs, which are all proanthocyanidin extracts, were isolated from Chinese bayberry leaves according to our previous study (6). The finely ground powder of the leaves was extracted with aqueous acetone [acetone:water, 80:20 (v/v)] containing $0.1 \%(\mathrm{w} / \mathrm{v})$ ascorbic acid at room temperature for $12 \mathrm{~h}$, and was then subjected to rotary-evaporation to remove the acetone. The aqueous phase was recovered and washed with hexane to remove nonpolar material, and the organic solvents were subsequently evaporated and lyophilized to obtain the bayberry leaf extracts.

To further purify the sample, a solution of the extracts, re-dissolved in $50 \%$ methanol, was loaded onto a column of Sephadex LH-20 and eluted stepwise with 50\% methanol to remove pigments and sugars, 90\% methanol to remove the majority of flavonoids, $50 \%$ acetone to elute the majority of proanthocyanidins and $70 \%$ acetone to clean the column. The fraction that was eluted by $50 \%$ acetone was used for subsequent analyses and was considered to consist of PCBLs.

Further separation of PCBLs according to polymerization degree was conducted using high-performance liquid chromatography (HPLC) on a Luna silica preparative column (Phenomenex, Torrance, CA, USA; $21.2 \mathrm{~mm}$ inner diameter $\mathrm{x} 250 \mathrm{~mm}$ ) with a $5-\mu \mathrm{m}$ particle size at $37^{\circ} \mathrm{C}$ using a hexane/methanol/ethyl acetate solution as the mobile phase. A Shimadzu preparative HPLC system (Shimadzu Corporation, Kyoto, Japan) equipped with a CBM-20A module, an SIL-10AP autosampler, an SPD-20A ultraviolet-visible detector and two LC-8A pumps were used. On each run, $1 \mathrm{ml}(200 \mathrm{mg} / \mathrm{ml})$ PCBL was applied (6). The fractions were collected manually when the target peak was visible on the screen and evaporated under a vacuum. According to our previous results (6), fractions containing dimers and trimers (N2-N7) were considered to be OPAs, which made up almost $23.9 \%$ of the PCBLs, whereas fractions containing tetramers and higher polymerizations of proanthocyanidins (N8-N10) were considered to be PPAs and composed almost $47.8 \%$ of the PCBLs. The remaining $~ 30 \%$ consisted of epigallocatechin gallate (EGCG), myricetin deoxyhexoside, myricetin deoxyhexoside-gallate, and other polyphenols not detected by normal-phase HPLC/electrospray ionization mass spectrometry.
Cell culture. OVCAR-3 human ovarian cancer cells were provided by Dr B. Jiang, Department of Microbiology, Immunology, and Cell Biology, West Virginia University (Morgantown, WV, USA). Cells were cultured in RPMI-1640 medium (Sigma-Aldrich, Merck Millipore, Darmstadt, Germany) supplemented with $10 \%$ fetal bovine serum (FBS; Invitrogen; Thermo Fisher Scientific, Inc., Waltham, MA, USA) at $37^{\circ} \mathrm{C}$ in a humidified incubator with $5 \% \mathrm{CO}_{2}$.

Cell viability assay. OVCAR-3 cells were seeded onto 96-well plates at a density of $1 \times 10^{4}$ cells/well $(100 \mu \mathrm{l})$ and incubated overnight at $37^{\circ} \mathrm{C}$ prior to treatment with 0 (control), 5, 10, 20, 40, 80 or $160 \mu \mathrm{g} / \mathrm{ml}$ of PCBL, OPA or PPA for $24 \mathrm{~h}$. A stock solution of each sample was prepared in dimethyl sulfoxide (DMSO) at $160 \mu \mathrm{g} / \mathrm{ml}$ and stored at $-20^{\circ} \mathrm{C}$. The varying concentrations of each sample were prepared in RPMI-1640 medium for cell treatments, and DMSO was included in the preparations to ensure equal concentrations of DMSO in each treatment. Control cells received an equal volume of DMSO only. Cell viability was analyzed with a CellTiter 96 Aqueous One Solution Cell Proliferation Assay (Promega Corporation, Madison, WI, USA), following the protocol of the manufacturer, and normalized to control wells for statistical analysis.

Apoptosis assessment by Hoechst 33342 staining. The OVCAR-3 cells were seeded in $60 \mathrm{~mm}$ dishes at a concentration of $6 \times 10^{5}$ cells/dish and incubated overnight at $37^{\circ} \mathrm{C}$. The cells were treated with $0,10,20,40$ or $80 \mu \mathrm{g} / \mathrm{ml}$ of PCBLs, OPAs and PPAs for $24 \mathrm{~h}$. Subsequent to treatment, the cells were stained with $10 \mu \mathrm{g} / \mathrm{ml}$ Hoechst 33342 (Sigma-Aldrich; Merck Millipore) in PBS for $10 \mathrm{~min}$ in the dark at $37^{\circ} \mathrm{C}$. Cell apoptosis was examined under a fluorescence microscope (Zeiss AG, Oberkochen, Germany). Apoptotic cells were considered to exhibit condensed or fragmented nuclei.

Flow cytometry. To quantify the induction of apoptotic death of ovarian cancer cells by PCBLs, OPAs and PPAs, annexin V and propidium iodide (PI) staining was performed, followed by flow cytometry as previously described (11). The cells were plated to $60 \%$ confluence overnight under the aforementioned conditions, and subsequently treated with 0,20 and $40 \mu \mathrm{g} / \mathrm{ml}$ PCBLs, OPAs and PPAs. After $24 \mathrm{~h}$ of treatment, the adherent cells were harvested by trypsinization, and adherent and nonadherent cells were collected using centrifugation at $1000 \mathrm{x} g$ for 5 min. Annexin V and PI staining was then performed on the cells using a Vybrant Apoptosis Assay kit 2 (Molecular Probes; Thermo Fisher Scientific Inc.) following the protocol provided by the manufacturer. Subsequent to staining, a flow cytometer (FACSCalibur ${ }^{\mathrm{TM}}$ system; BD Biosciences, San Jose, CA, USA) was used for the quantification of the apoptotic cells.

Caspase-3/7, -8 and -9 assays. Cellular caspase-3/7, -8 and -9 activities were measured with Caspase-Glo Assay kits (Promega Corporation). The assays provide a proluminescent substrate that is cleaved to aminoluciferin by caspase-3/7, -8 or -9 . The released aminoluciferin substrate reacts with luciferase, generating a luminescent signal, which is proportional to the activity of the caspases. To perform the assay, OVCAR-3 cells were seeded into 96-well plates at a density of $1 \times 10^{4}$ cells/well and incubated overnight at $37^{\circ} \mathrm{C}$. The cells 
were treated with $0,5,10,20$ or $40 \mu \mathrm{g} / \mathrm{ml}$ of PCBLs, OPAs or PPAs for $24 \mathrm{~h}$, before the plates containing the cells were removed from the incubator and allowed to equilibrate to room temperature for $30 \mathrm{~min}$. Caspase-Glo reagent $(100 \mu \mathrm{l})$ was then added to each well, and the plates were incubated at room temperature for $2 \mathrm{~h}$. The luminescence of each sample was measured in a Synergy HT Multi-Mode Microplate Reader (BioTek Instruments, Inc., Winooski, VT, USA).

Western blot analysis. Ovarian cancer cells $\left(6 \times 10^{5}\right.$ cells $)$ were seeded in $60-\mathrm{mm}$ dishes, incubated overnight at $37^{\circ} \mathrm{C}$, and treated with 0,10 or $20 \mu \mathrm{g} / \mathrm{ml}$ of PCBLs, OPAs or PPAs for $24 \mathrm{~h}$. The cells were harvested and lysed with M-PER Mammalian Protein Extraction Reagent (Pierce; Thermo Fisher Scientific, Inc.) supplemented with Halt Protease and Phosphatase Inhibitor Single-Use Cocktail (Pierce; Thermo Fisher Scientific, Inc.) according to the protocol of the manufacturer. Total protein levels were assayed with a Bicinchoninic Acid Protein Assay kit (Pierce; Thermo Fisher Scientific, Inc.). The cell lysates were separated by $10 \%$ SDS-PAGE and blotted onto a nitrocellulose membrane with a Mini-Protean 3 System (Bio-Rad Laboratories, Inc., Hercules, CA, USA). The membranes were then blocked in 5\% nonfat milk in TBS containing $0.1 \%$ Tween-20 (TBST) for $1 \mathrm{~h}$ at room temperature and incubated with indicated primary antibodies overnight at $4^{\circ} \mathrm{C}$ followed by horseradish peroxidase (HRP)-conjugated secondary antibodies for $2 \mathrm{~h}$ at $37^{\circ} \mathrm{C}$. Antibodies against caspase-3 (cat. no. 9662), caspase-8 (cat. no. 9746), caspase-9 (cat.no. 9508), p53 upregulated modulator of apoptosis (PUMA; cat. no. 12450), B-cell lymphoma 2 (Bcl-2; cat. no. 3498), FAS (cat. no. 8023), Fas L (cat. no. 4273), Fas-associated protein with death domain (FADD; cat. no. 2782), p21 (cat. no. 2947), anti-mouse IgG with HRP-conjugated secondary antibody (cat. no. 7076) and anti-rabbit IgG with HRP-conjugated secondary antibody (cat. no. 7074) were purchased from Cell Signaling Technology, Inc. (Danvers, MA, USA) and all were used at a dilution of 1:1,000. Antibodies against Bcl-extra large (xL; cat. no. 136207), Bcl-2-associated X protein (Bax; cat. no. 4239), Bcl-2-associated death promoter (Bad; cat. no. 4702), DR4 (cat. no. 65312), DR5 (cat. no. 65314), PTEN (cat. no. 7974), p53 (cat. no. 47698), MDM2 (cat. no. 812) and GAPDH (cat. no. 47724) were purchased from Santa Cruz Biotechnology Inc. (Dallas, TX, USA) and all were used at a dilution of 1:200. Subsequent to washing with TBST, the antigen-antibody complex was visualized with the SuperSignal West Dura Extended Duration Substrate (Pierce; Thermo Fisher Scientific, Inc.). The protein bands were detected and quantitated with ChemiDoc XRS+ System and Image Lab Software (Bio-Rad Laboratories, Inc., version 5.1) and normalized to the corresponding GAPDH level for analysis.

Transfection with small interfering RNA (siRNA). The ovarian cancer OVCAR-3 cells (6x $10^{5}$ cells) were seeded in $60-\mathrm{mm}$ dishes, incubated overnight at $37^{\circ} \mathrm{C}$, and transfected with p53 siRNA (Santa Cruz Biotechnology, Inc.) using Lipofectamine ${ }^{\circledR} 2000$ transfection reagent (Invitrogen; Thermo Fisher Scientific, Inc.) according to the protocol of the manufacturer. Following a 24-h transfection period, the cells were treated with OPAs or PPAs for $24 \mathrm{~h}$. The cell lysates were collected for western blot analysis.
Statistical analysis. Data are presented as the mean \pm standard deviation of three independent experiments. Statistical significance was determined with SPSS 18.0 (SPSS, Inc., Chicago, IL, USA) by one-way analysis of variance followed by the Duncan multiple range test. $\mathrm{P}<0.05$ was considered to indicate a statistically significant difference.

\section{Results}

PCBLs, OPAs and PPAs inhibit proliferation and induce apoptosis in OVCAR-3 human ovarian cancer cells. The treatment of OVCAR-3 cells with $0-100 \mu \mathrm{g} / \mathrm{ml}$ PCBLs, OPAs or PPAs resulted in significant concentration-dependent reductions in cell proliferation, with half-maximal inhibitory concentrations $\left(\mathrm{IC}_{50}\right)$ of 82.32, 34.98 and $15.11 \mu \mathrm{g} / \mathrm{ml}$, respectively (Fig. 1A). PPAs induced a greater level of cytotoxicity in OVCAR-3 cells compared with the OPAs.

To determine whether the decrease in cell viability was due to apoptotic cell death, the alterations in cellular and nuclear morphology of OVCAR-3 cells following their treatment with PCBLs, OPAs or PPAs $(0-80 \mu \mathrm{g} / \mathrm{ml})$ for $24 \mathrm{~h}$ were assessed using Hoechst 33342 DNA staining and fluorescence microscopy (Fig. 1B). Following each of the treatments, numerous apoptotic cells, exhibiting condensed or fragmented nuclei, were observed, and this effect was concentration-dependent. As presented in Fig. 1B, OPAs and PPAs significantly increased the percentage of apoptotic cells at $20 \mu \mathrm{g} / \mathrm{ml}(\mathrm{P}<0.05)$, while an equivalent effect was observed in cells treated with $80 \mu \mathrm{g} / \mathrm{ml}$ PCBLs, which was in accordance with the cell viability results.

The rates of apoptosis in OVCAR-3 cells subsequent to PCBL, OPA or PPA treatment were determined by flow cytometry. The apoptotic cells were categorized as early or late apoptotic cells, which are presented in the lower right and upper right quadrants of the fluorescence-activated cell sorting histograms, respectively (Fig. 2A). PCBLs, OPAs and PPAs induced a significant concentration-dependent induction of apoptosis at the early and late stages of apoptosis following $24 \mathrm{~h}$ of treatment $(\mathrm{P}<0.05$; Fig. $2 \mathrm{~B})$. The rates of total apoptosis increased from $4.22 \pm 1.73 \%$ in untreated control cells to $13.57 \pm 0.90,21.47 \pm 2.44$ and $27.83 \pm 0.32 \%$ in cells treated with PCBLs, OPAs and PPAs at $40 \mu \mathrm{g} / \mathrm{ml}$, respectively.

PCBL, OPA and PPA-induced apoptosis is caspase-dependent in OVCAR-3 cells. Apoptosis may be initiated through an extrinsic pathway, which is associated with the activation of caspase-8, or an intrinsic pathway, which is associated with the activation of caspase-9. These two pathways converge on the activation of caspase-3/7 and trigger apoptosis (12). In order to investigate the induction of apoptosis by PCBLs, OPAs and PPAs, the enzymatic activity levels of caspase- $-3 / 7,-8$ and -9 were evaluated using Caspase-Glo Assay kits and western blot analysis in OVCAR-3 cells.

As presented in Fig. 3A, treatment with $20 \mu \mathrm{g} / \mathrm{ml}$ PCBLs, $10 \mu \mathrm{g} / \mathrm{ml}$ OPAs or $20 \mu \mathrm{g} / \mathrm{ml}$ PPAs maximally increased the caspase-3/7 enzymatic activity levels to $1.82-, 1.88$ - and 2.15 -fold of the levels of the control group $(0 \mu \mathrm{g} / \mathrm{ml})$, respectively $(\mathrm{P}<0.05)$. With regard to the caspase- 8 enzymatic activity, treatment with $10 \mu \mathrm{g} / \mathrm{ml}$ PCBLs, $20 \mu \mathrm{g} / \mathrm{ml}$ OPAs and $40 \mu \mathrm{g} / \mathrm{ml}$ PPAs maximally increased the levels to 1.46-, 1.87- and 1.96-fold of the control levels, respectively $(\mathrm{P}<0.05$; 


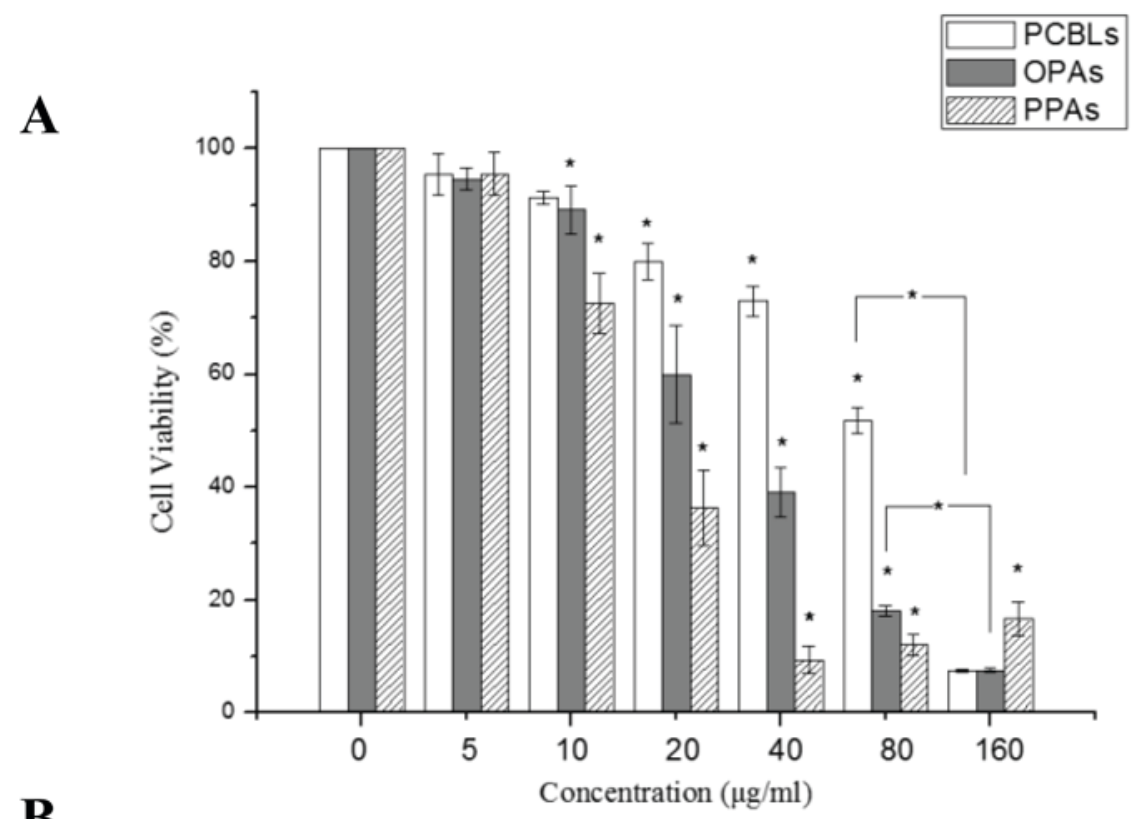

B

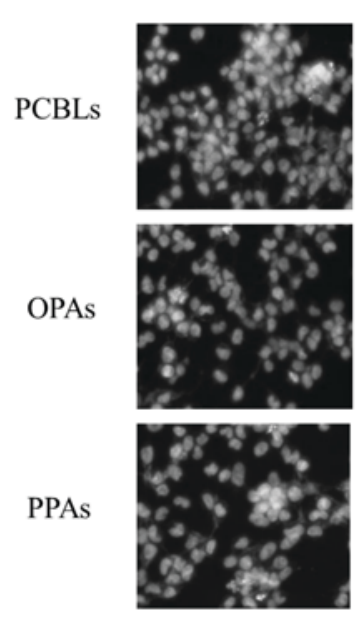

10
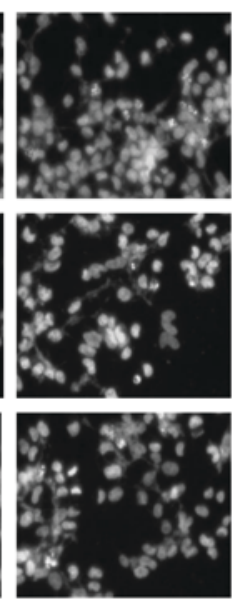

20
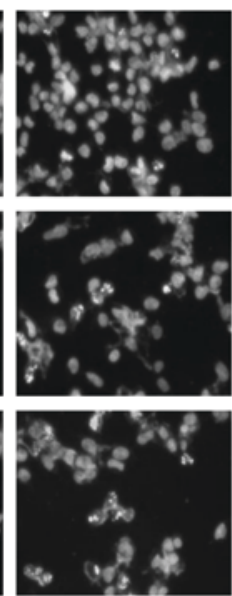

40
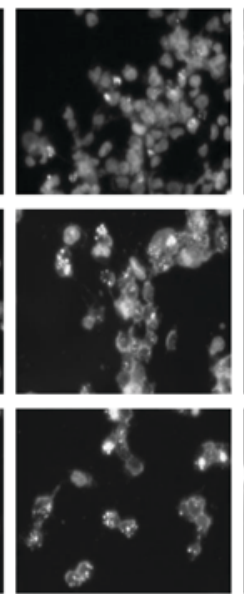

80
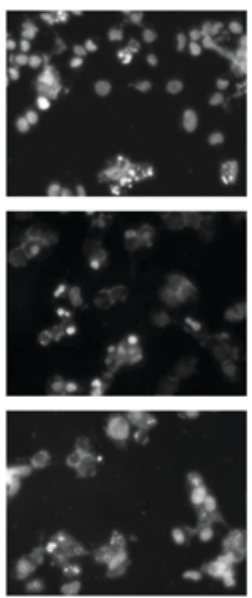

Figure 1. PCBLs, OPAs and PPAs decrease cell viability and increase apoptosis of OVCAR-3 human ovarian cancer cells. (A) Cell viability was determined using a tetrazolium dye assay. (B) Hoechst 33342 staining of OVCAR-3 cells was detected by fluorescence microscopy (magnification, $\mathrm{x} 400$ ). ${ }^{*} \mathrm{P}<0.05$ compared with the control, or between specific groups. PCBL, Chinese bayberry leaf prodelphinidin extract; OPA, oligomeric proanthocyanidin; PPA, polymeric proanthocyanidins.

Fig. 3B). As shown in Fig. 3C, treatment with $40 \mu \mathrm{g} / \mathrm{ml}$ PCBLs, $10 \mu \mathrm{g} / \mathrm{ml}$ OPAs and $40 \mu \mathrm{g} / \mathrm{ml}$ PPAs, maximally increased the caspase-9 enzymatic activity to 1.50-, 1.81- and 2.09-fold of the control levels, respectively $(\mathrm{P}<0.05)$.

Western blotting indicated that the PCBLs, OPAs and PPAs decreased the expression levels of procaspase-3, procaspase- 8 and procaspase- 9 in a concentration-dependent manner (Fig. 3D). In addition, the level of cleaved caspase-3 increased, which was in accordance with the aforementioned Caspase-Glo results, and OPAs and PPAs also increased the expression levels of cleaved caspase- 8 . These findings suggest that PCBLs, OPAs and PPAs induce caspase-dependent apoptosis.

Effect of PCBLs, OPAs and PPAs on the intrinsic apoptotic pathway. To clarify whether the intrinsic pathway was involved in PCBL, OPA and PPA-induced apoptosis, western blot analysis was used to examine the protein expression levels of various proapoptotic Bcl-2 family proteins, including PUMA, Bax and Bcl-2-associated death promoter (Bad). The antiapoptotic Bcl-2 family proteins Bcl-2 and Bcl-extra large (xL) were also assessed (Fig. 4). PCBLs, OPAs and PPAs increased the expression levels of PUMA, Bax and Bad. In addition, an inhibitory effect of PCBLs, OPAs and PPAs on Bcl-xL was observed. These findings suggest that PCBLs, OPAs and PPAs can induce apoptosis in OVCAR-3 cells via the intrinsic apoptotic pathway.

Effect of PCBLs, OPAs and PPAs on the extrinsic apoptotic pathway. The present study subsequently investigated whether the PCBLs, OPAs and PPAs induced apoptosis via the extrinsic apoptotic pathway. As presented in Fig. 5, PCBLs only induced Fas expression, whereas OPAs and PPAs increased the expression of Fas and death receptor (DR) 5 proteins (48 kDa). PPAs also exerted a stimulatory effect on FADD protein. PCBLs exhibited no effect on DR4, DR5, FasL or FADD expression. 
$\mu \mathrm{g} / \mathrm{ml}$

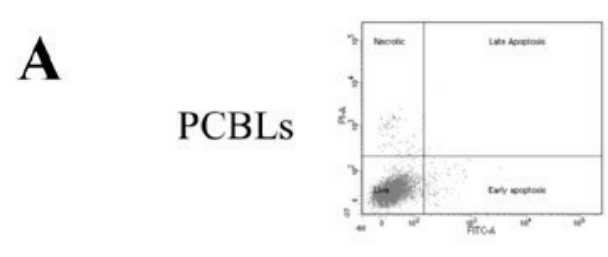

OPAs

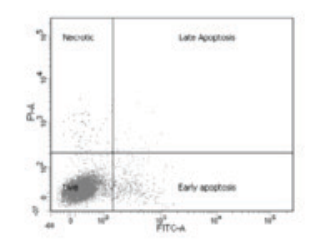

PPAs

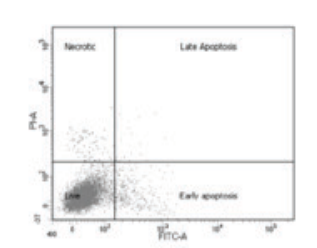

20
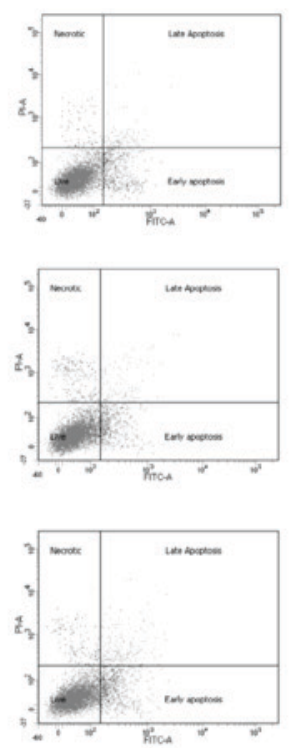

40
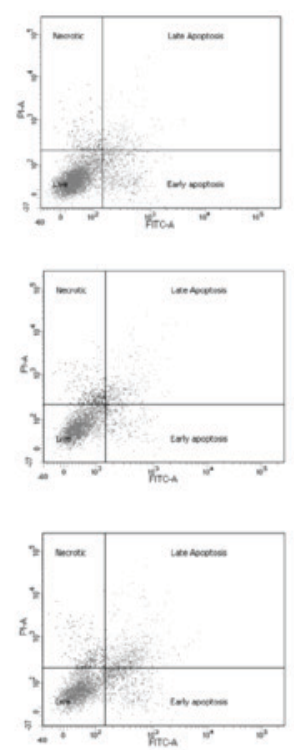

\section{B}

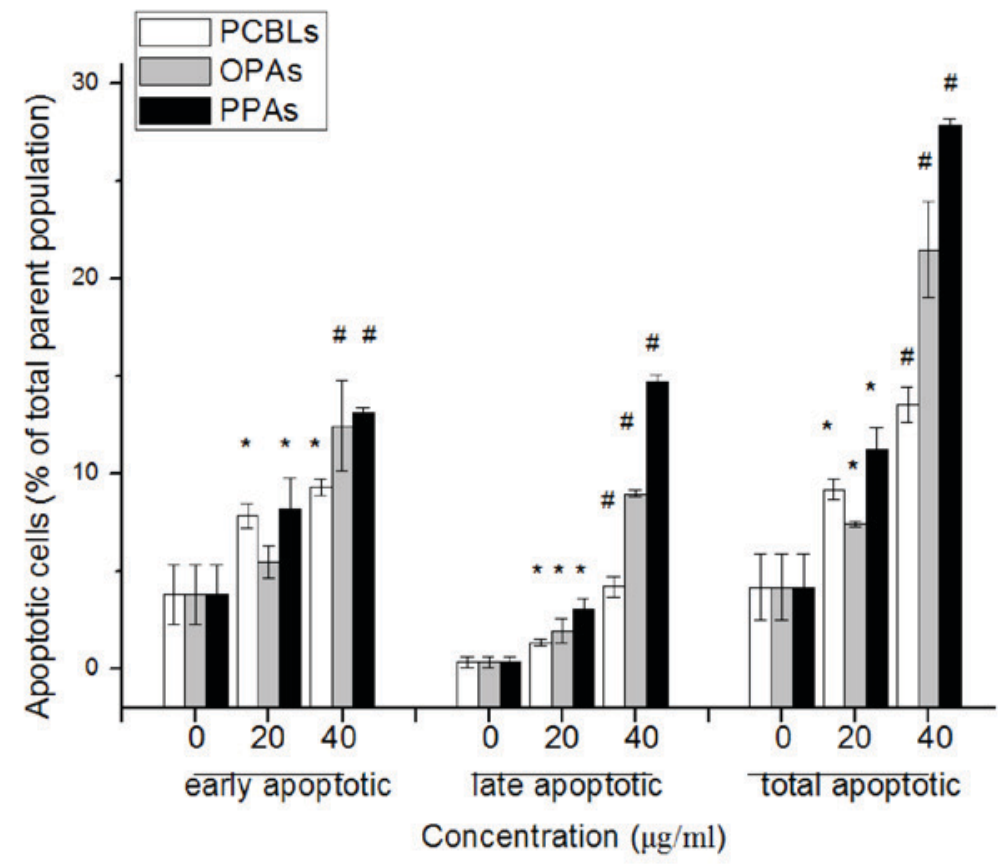

Figure 2. PCBLs, OPAs and PPAs increase apoptosis of OVCAR-3 human ovarian cancer cells. (A) Flow cytometric analysis was used to assess cell apoptosis rates following annexin V/propodium iodide double labeling. (B) The histogram indicates the rates of apoptosis in the cells (\%). Data is presented as the mean \pm standard deviation from 3 independent experiments. ${ }^{*} \mathrm{P}<0.05$ vs. $0 \mu \mathrm{g} / \mathrm{ml},{ }^{*} \mathrm{P}<0.05$ vs. $20 \mu \mathrm{g} / \mathrm{ml}$. PCBL, Chinese bayberry leaf prodelphinidin extract; OPA, oligomeric proanthocyanidin; PPA, polymeric proanthocyanidins.

As an increase in Fas expression occurred subsequent to PCBL, OPA or PPA treatment, the results suggest that PCBLs, OPAs and PPAs may induce apoptosis in OVCAR-3 cells through a Fas-associated extrinsic pathway.

Role of p53 in PCBL-, OPA- and PPA-induced apoptosis. p53 is crucial in the induction of apoptosis in human and murine cells following DNA damage (13). Although OVCAR-3 cells harbor a point mutation in the $\mathrm{p} 53$ gene, which results in single amino acid changes, p53 still serves an important role in the apoptosis and cell cycle arrest of OVCAR-3 cells induced by certain cytokines and compounds (14-16). p53 levels are primarily controlled by the proto-oncogene product mouse double minute 2 (MDM2), which ubiquitinates p53 and facilitates the degradation of the protein by proteasomes (17). p21 is a p53 transcription target involved in the major functions of tumor suppressor cell cycle arrest and apoptosis (18).

As presented in Fig. 6A, the results of the current study revealed that OPAs and PPAs stimulated p53, MDM2 and p21 expression in a concentration-dependent manner, while PCBLs exhibited no effect on these proteins. In order to clarify the effect of p53 on the induction of apoptosis by OPAs and PPAs, a p53-specific siRNA was used. The results presented in Fig. 6B indicated that the knockdown of p53 by the siRNA $(50 \mathrm{nM})$ resulted in the inhibition of p53 expression subsequent to OPA and PPA treatment $(0-20 \mu \mathrm{g} / \mathrm{ml})$. 

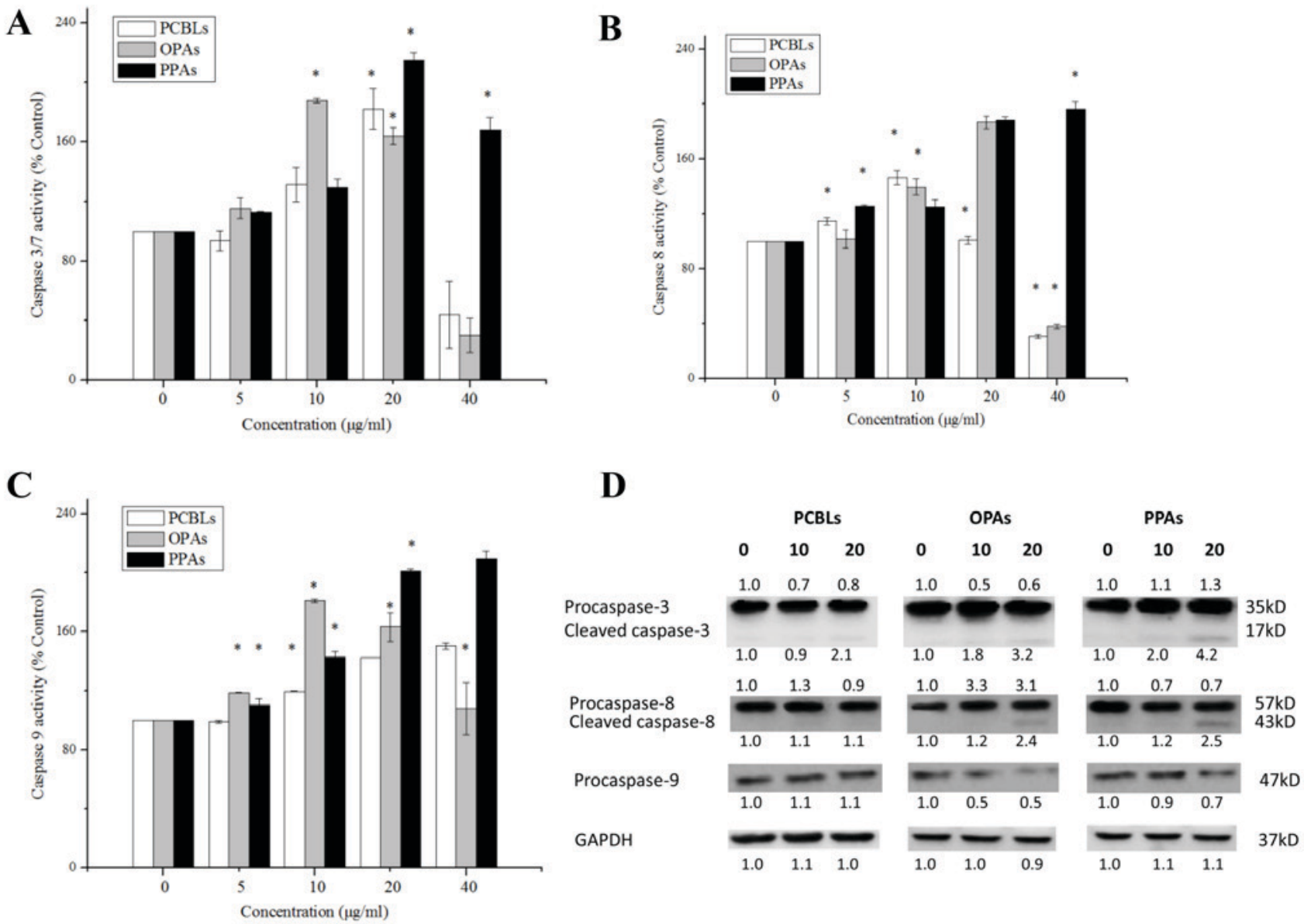

Figure 3. Activation of caspases during PCBL, OPA and PPA-induced apoptosis in OVCAR-3. (A) Caspase-3/7, (B) caspase-8 and (C) caspase-9 activity levels were assessed following treatment with PCBLs, OPAs and PPAs for $24 \mathrm{~h}$. The caspase activity of the control cells subsequent to treatment $(0 \mu \mathrm{g} / \mathrm{ml}) \mathrm{was}$ set as $100 \%$. Data represents the mean \pm standard deviation from 3 independent experiments. ${ }^{*} \mathrm{P}<0.05$ compared with the control. (D) Protein expression levels of caspase-3/7, -8 and -9 were assessed by western blot analysis. Protein expression levels were measured by densitometry and the values above and below the blots indicate the relative ratios. Results are representative of $\geq 2$ independent experiments. PCBL, Chinese bayberry leaf prodelphinidin extract; OPA, oligomeric proanthocyanidin; PPA, polymeric proanthocyanidin.

PCBLS

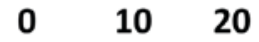

Puma

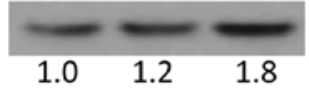

$\mathrm{Bcl}-2$

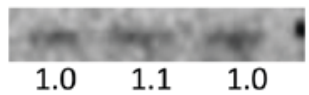

$\mathrm{BCl}-\mathrm{xL}$

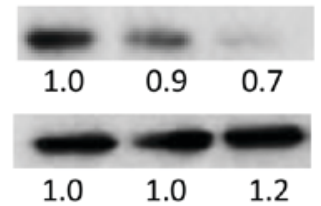

Bad

GAPDH

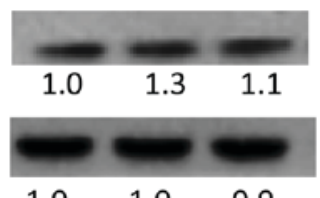

OPAs

$10 \quad 20$
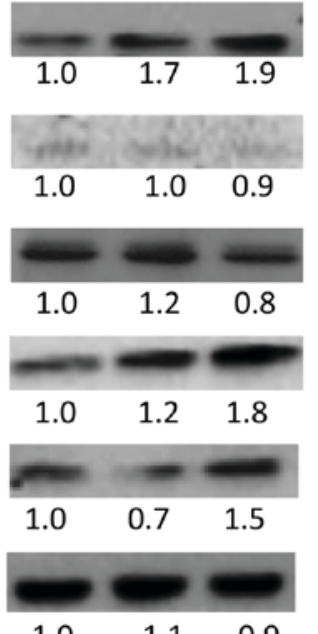

PPAs
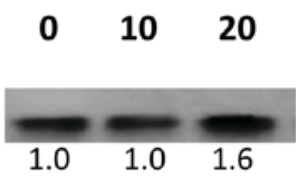

$23 \mathrm{kD}$

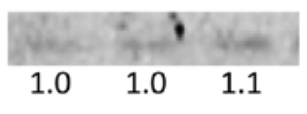

$28 \mathrm{kD}$

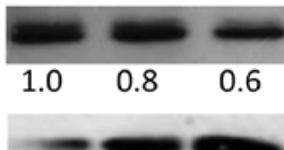

$30 \mathrm{kD}$

$20 \mathrm{kD}$

$\begin{array}{lll}1.0 & 2.3 \quad 3.6\end{array}$

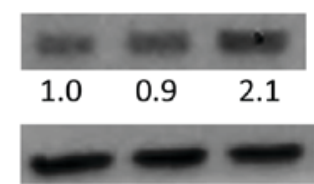

$25 \mathrm{kD}$

$37 \mathrm{kD}$

Figure 4. PCBLs, OPAs and PPAs induce intrinsic apoptosis in ovarian cancer cells. Results are representative of $\geq 2$ independent experiments that exhibited similar patterns. Protein expression levels were measured by densitometry and the values above and below the blots indicate the relative ratios. PCBL, Chinese bayberry leaf prodelphinidin extract; OPA, oligomeric proanthocyanidin; PPA, polymeric proanthocyanidin. Puma, p53-upregulated modulator of apoptosis; Bcl-2, B-cell lymphoma-2; Bcl-xL, Bcl-extra large; Bax, Bcl-2-associated X protein; Bad, Bcl-2-associated death promoter. 


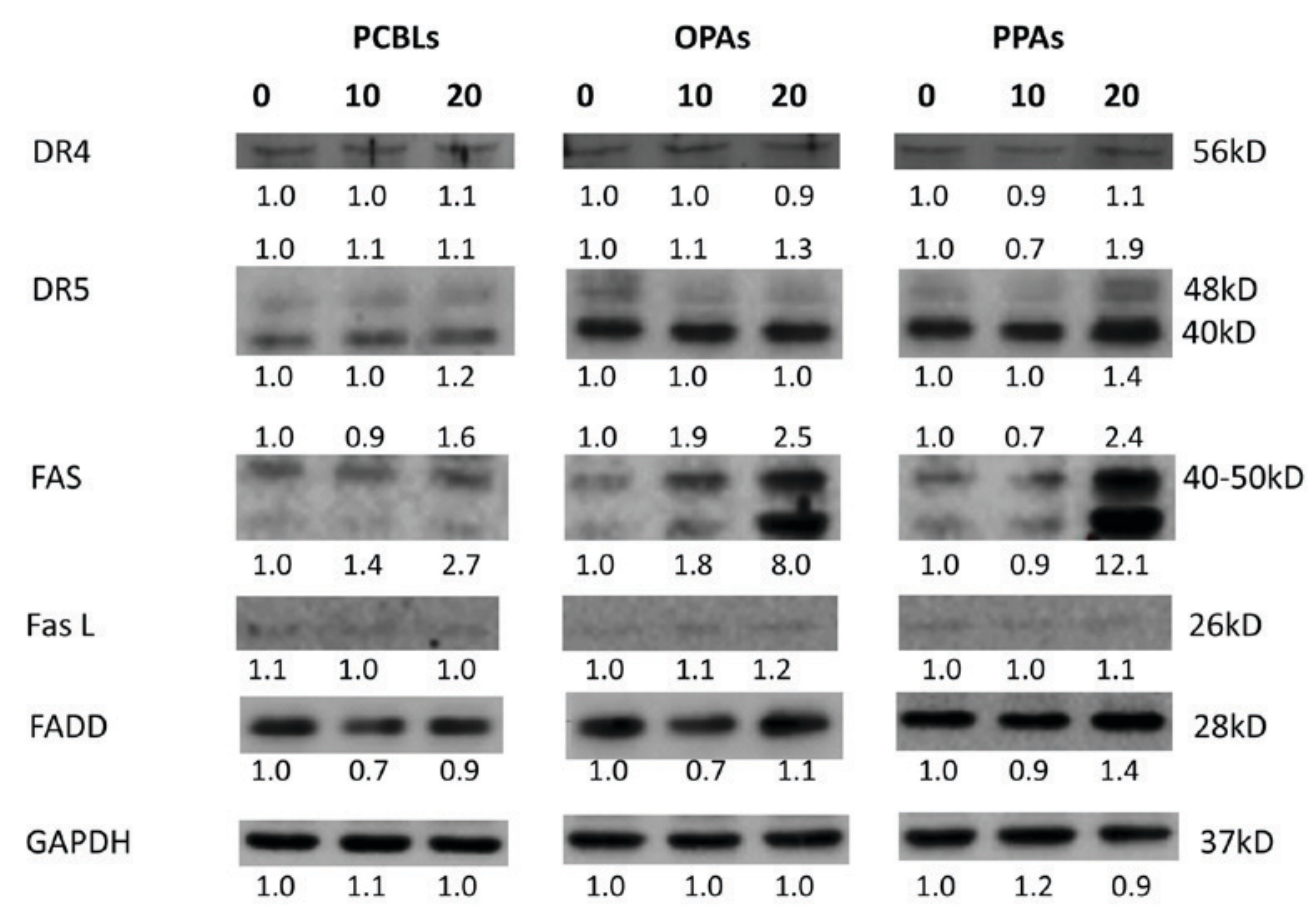

Figure 5. PCBLs, OPAs and PPAs induce extrinsic apoptosis in ovarian cancer cells. Results are representative of $\geq 2$ independent experiments that showed similar patterns. Protein expression levels were measured by densitometry and the values above and below the blots indicate the relative ratios. PCBL, Chinese bayberry leaf prodelphinidin extract; OPA, oligomeric proanthocyanidin; PPA, polymeric proanthocyanidin.

The depletion of p53 led to an associated decrease in the expression of p21, DR5, Fas, PUMA and phosphatase and tensin homolog proteins. These observations suggest that $\mathrm{p} 53$ is a critical mediator of OPA- and PPA-induced apoptosis in OVCAR-3 cells.

\section{Discussion}

To the best of our knowledge, the present study was the first to examine the apoptotic effect of PCBLs. According to our previous studies, PCBLs are almost entirely of the galloylated-prodelphinidin type, which is unusual in the plant kingdom as plants normally contain procyanidins and a few prodelphins $(6,7)$. The structural units of prodelphinidins in Chinese bayberry leaves are epicatechin (EC), epicatechin gallate (ECG), epigallocatechin (EGC) and EGCG $(6,7)$. OPAs are fractions collected from preparative HPLC, which mainly contain dimers and trimers including $\mathrm{EGC}+\mathrm{ECG}$, $\mathrm{EGC}+\mathrm{EGCG}, 2 \mathrm{EGCG}, 2 \mathrm{ECG}, 2 \mathrm{EC}+\mathrm{EGC}, 2 \mathrm{EGC}+\mathrm{EGCG}$ and 3EGCG. PPAs are fractions composed of proanthocyanidin tetramers or polymers of a higher polymerization degree, including 2EGC+2EGCG, EGC+3EGCG, 3ECG+EGCG, 4EGCG and numerous other unidentified proanthocyanidins. OPAs constitute $23.9 \%$ of PCBLs, whereas PPAs constitute $\sim 47.8 \%$.

The present study demonstrated that PCBLs, OPAs and PPAs induced apoptosis in a concentration-dependent manner in OVCAR-3 cells, a finding that was confirmed by Hoechst 33342 DNA staining and annexin V and PI staining. The results revealed that PCBLs, OPAs and PPAs led to early and late apoptosis in cells in a concentration-dependent manner. According to the total apoptotic cell percentage, PPAs were more effective than OPAs for inducing apoptosis.
Based on the observed increase in apoptosis in the treated cells, the next aim of the present study was to examine the involvement of caspases, which have major roles in the execution of apoptotic events. Therefore, the levels of active caspases were investigated by Caspase-Glo assay in cell lysates. The experiments of the present study revealed that treatment of cells with 20 or $40 \mu \mathrm{g} / \mathrm{ml}$ PPAs was associated with the most prominent increase in the activation of caspase-3/7, -8 and -9 . The results indicated that PCBLs, OPAs and PPAs exerted a strong and concentration-dependent effect on the induction of active caspase-3/7, -8 and -9 levels.

The majority of caspase-dependent apoptosis is associated with the mitochondrial pathway (9). The present study demonstrated that PCBLs, OPAs and PPAs increased the protein expression of the proapoptotic Bcl-2 family proteins PUMA, Bax and BAD, and decreased the protein expression of the anti-apoptotic Bcl-2 family protein Bcl-xL (Fig. 4). However, due to the low expression level of Bcl-2, an inhibitory effect on this protein was not observed. PCBLs, OPAs and PPAs were also observed to affect the extrinsic pathway, primarily via increases in DR5 and Fas expression levels.

Grape seed extract (GSE) is one of the most well known nutrition supplements containing proanthocyanidins. A number of studies have demonstrated that grape seed procyanidins induce apoptosis in cancer cells through the intrinsic and extrinsic pathways by downregulating antiapoptotic proteins and upregulating several proapoptotic factors. This eventually leads to the activation of caspases-9 and -3 (19-21), which is in accordance with the results of the present study. The present study also showed that p53 is associated with OPA- and PPA-induced apoptosis (Fig. 6). However, several studies have found that the cytotoxic effect exhibited by overall GSE is independent of the p53 status of the cancer cell lines $(22,23)$. 

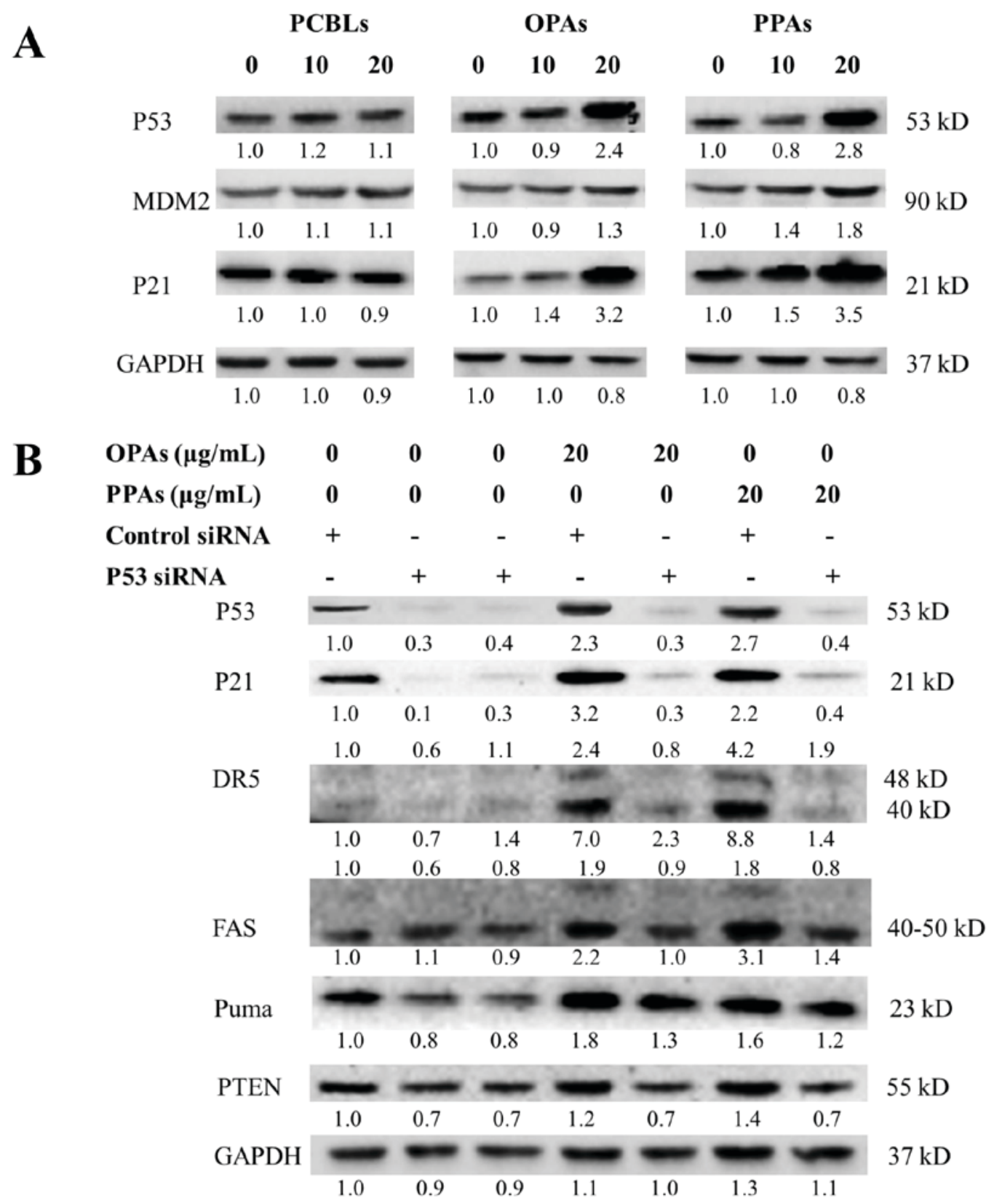

Figure 6. p53 is associated with OPA- and PPA-induced apoptosis in OVCAR-3 cells. (A) Effects of PCBLs, OPAs and PPAs on the protein expression of p53, MDM2 and p21 were determined by western blot analysis. (B) Effect of p53 siRNA (50 nM) on the protein expression of p21, DR5, Fas, PUMA and PTEN were determined by western blot analysis. Results are representative of $\geq 2$ independent experiments that showed similar patterns. Protein expression levels were measured by densitometry and the values above and below the blots indicate the relative ratios. PCBL, Chinese bayberry leaf prodelphinidin extract; OPA, oligomeric proanthocyanidin; PPA, polymeric proanthocyanidin; MDM2, mouse double minute 2 homolog; PTEN, phosphate and tensin homolog; si, small interfering; PUMA, p53-upregulated modulator of apoptosis; DR5, death receptor 5.

In the present study OPAs and PPAs exhibited strong effects on OVCAR-3 cell proliferation and apoptosis. Several studies have reported evidence that monomeric catechins and proanthocyanidin monomers, dimers and trimers can be absorbed through human intestinal Caco-2 epithelial cells $(24,25)$. However, the results of the present study revealed that PPAs (prodelphinidin tetramers and higher polymerizations) exerted greater cytotoxic and apoptotic activities compared with OPAs (dimers and trimers) in general. Similar results were observed by Miura et al (8), who separated eight procyanidin fractions according to the degree of polymerization using normal-phase chromatography, and detected strong antiproliferative activity of the procyanidin pentamers and higher degree fractions in B16 and BALB-MC.E12 cells.

Although the precise effect of the degree of polymerization remains unclear, diversity in prodelphinin stereochemistry, structure, molecular size, polarity and solubility may affect the bioavailability of prodelphinidins. Additional investigation into bioavailability, including analysis of metabolites and distribution, are required to clarify the anticancer activity of prodelphinidins, particularly polymers higher than tetramers, following oral administration in vivo. 


\section{Acknowledgements}

The authors would like to thank Dr Kathy Brundage from the Flow Cytometry Core at West Virginia University for providing technical help regarding cell apoptosis, Dr Haizhi Huang for expert technical assistance, and Mrs. Amy Mason-Hopkins for assistance with writing the study. The present study was supported by grants from the West Virginia Experimental Program to Stimulate Competitive Research (grant no. EPS-1003907) and the National Institutes of Health (grant nos. P20RR016477 and P20GM103434) awarded to the West Virginia IDeA Network of Biomedical Research Excellence. The present study was also supported by the Zhejiang Province Sci-tech Special Commissioner Earmarks (grant no. 2012T2T123).

\section{References}

1. Nandakumar V, Singh T and Katiyar SK: Multi-targeted prevention and therapy of cancer by proanthocyanidins. Cancer Lett 269: 378-387, 2008.

2. Prior RL and Gu L: Occurrence and biological significance of proanthocyanidins in the American diet. Phytochemistry 66: 2264-2280, 2005.

3. de la Iglesia R, Milagro FI, Campión J, Boqué N and Martínez JA: Healthy properties of proanthocyanidins. Biofactors 36: 159-168, 2010.

4. Chen K, Xu C, Zhang B and Ferguson IB: Red bayberry: Botany and horticulture. Horticultural Rev 30: 83-114, 2004.

5. Zhang SM, Gao ZS, Xu CJ and Chen KS: Genetic diversity of Chinese bayberry (Myrica rubra Sieb. et Zucc.) accessions revealed by amplified fragment length polymorphism. Hort Sci 44: 487-491, 2009.

6. Fu Y, Qiao L, Cao Y, Zhou X, Liu Y and Ye X: Structural elucidation and antioxidant activities of proanthocyanidins from Chinese bayberry (Myrica rubra Sieb. et Zucc.) leaves. PLoS One 9: e96162, 2014.

7. Yang H, Ye X, Liu D, Chen J, Zhang J, Shen Y and Yu D: Characterization of unusual proanthocyanidins in leaves of bayberry (Myrica rubra Sieb. et Zucc.). J Agric Food Chem 59: 1622-1629, 2011.

8. Miura T, Chiba M, Kasai K, Nozaka H, Nakamura T, Shoji T, Kanda T, Ohtake Y and Sato T: Apple procyanidins induce tumor cell apoptosis through mitochondrial pathway activation of caspase-3. Carcinogenesis 29: 585-593, 2008.

9. Han MH, Lee WS, Jung JH, Jeong JH, Park C, Kim HJ, Kim G, Jung JM, Kwon TK, Kim GY, et al: Polyphenols isolated from Allium cepa L. induces apoptosis by suppressing IAP-1 through inhibiting PI3K/Akt signaling pathways in human leukemic cells. Food Chem Toxicol 62: 382-389, 2013.

10. Dai GH, Meng GM, Tong YL, Chen X, Ren ZM, Wang K and Yang F: Growth-inhibiting and apoptosis-inducing activities of Myricanol from the bark of Myrica rubra in human lung adenocarcinoma A549 cells. Phytomedicine 21: 1490-1496, 2014
11. Kaur M, Mandair R, Agarwal R and Agarwal C: Grape seed extract induces cell cycle arrest and apoptosis in human colon carcinoma cells. Nutr Cancer 60 (Suppl 1): S2-S11, 2008.

12. Kroemer G and Martin SJ: Caspase-independent cell death. Nat Med 11: 725-730, 2005.

13. Huang C, Ma WY, Goranson A and Dong Z: Resveratrol suppresses cell transformation and induces apoptosis through a p53-dependent pathway. Carcinogenesis 20: 237-242, 1999.

14. Guan YQ, Li Z, Yang A, Huang Z, Zheng Z, Zhang L, Li L and Liu JM: Cell cycle arrest and apoptosis of OVCAR-3 and MCF-7 cells induced by co-immobilized TNF- $\alpha$ plus IFN- $\gamma$ on polystyrene and the role of p53 activation. Biomaterials 33: 6162-6171, 2012.

15. Lin HY, Delmas D, Vang O, Hsieh TC, Lin S, Cheng GY, Chiang HL, Chen CE, Tang HY, Crawford DR, et al: Mechanisms of ceramide-induced COX-2-dependent apoptosis in human ovarian cancer OVCAR-3 cells partially overlapped with resveratrol. J Cell Biochem 114: 1940-1954, 2013.

16. Li B, Gao Y, Rankin GO, Rojanasakul Y, Cutler SJ, Tu Y and Chen YC: Chaetoglobosin K induces apoptosis and G2 cell cycle arrest through p53-dependent pathway in cisplatin-resistant ovarian cancer cells. Cancer Lett 356: 418-433, 2015.

17. Yang X, Fraser M, Moll UM, Basak A and Tsang BK: Akt-mediated cisplatin resistance in ovarian cancer: Modulation of p53 action on caspase-dependent mitochondrial death pathway. Cancer Res 66: 3126-3136, 2006.

18. Xia M, Knezevic D and Vassilev LT: p21 does not protect cancer cells from apoptosis induced by nongenotoxic p53 activation. Oncogene 30: 346-355, 2011.

19. Hsu CP, Lin YH, Chou CC, Zhou SP, Hsu YC, Liu CL, Ku FM and Chung YC: Mechanisms of grape seed procyanidin-induced apoptosis in colorectal carcinoma cells. Anticancer Res 29: 283-289, 2009.

20. Singh T, Sharma SD and Katiyar SK: Grape proanthocyanidins induce apoptosis by loss of mitochondrial membrane potential of human non-small cell lung cancer cells in vitro and in vivo. PLoS One 6: e27444, 2011.

21. Dinicola S, Cucina A, Antonacci D and Bizzarri M: Anticancer effects of grape seed extract on human cancers: A review. J Carcinogenesis Mutagenesis 8: 60-70, 2014.

22. Dinicola S, Cucina A, Pasqualato A, D'Anselmi F, Proietti S, Lisi E, Pasqua G, Antonacci D and Bizzarri M: Antiproliferative and apoptotic effects triggered by Grape Seed Extract (GSE) versus epigallocatechin and procyanidins on colon cancer cell lines. Int J Mol Sci 13: 651-664, 2012.

23. Prasad R and Katiyar SK: Bioactive phytochemical proanthocyanidins inhibit growth of head and neck squamous cell carcinoma cells by targeting multiple signaling molecules. PLoS One 7: e46404, 2012.

24. Deprez S, Mila I, Huneau JF, Tome D and Scalbert A: Transport of proanthocyanidin dimer, trimer, and polymer across monolayers of human intestinal epithelial Caco-2 cells. Antioxid Redox Signal 3: 957-967, 2001.

25. Faria A, Mateus N, de Freitas V and Calhau C: Modulation of MPP+ uptake by procyanidins in Caco-2 cells: Involvement of oxidation/reduction reactions. FEBS Lett 580: 155-160, 2006. 